\title{
MYIASIS HUMAIN : CAS CLINIQUE
}

\section{ARTICLE ORIGINAL}

SILVA, Agnaldo Plácido da¹, PLÁCIDO, Eloá Jessica Mendes dos Santos², MORAES, Walber Breno de Souza ${ }^{3}$

SILVA, Agnaldo Plácido da. PLÁCIDO, Eloá Jessica Mendes dos Santos. MORAES, Walber Breno de Souza. Myiasis humain : Cas clinique. Revista Científica Multidisciplinar Núcleo do Conhecimento. An 05, Ed. 08, vol. 10, p. 39 à 46 . août 2020.

ISSN:

2448-0959,

Lien

d'accès:

https://www.nucleodoconhecimento.com.br/sante/myiasis-humain,

DOI:

10.32749/nucleodoconhecimento.com.br/sante/myiasis-humain

\section{RÉSUMÉ}

La myiase est considérée comme une infestation de dermatozoose causée par l'infestation de larves de diptères dans les tissus ou les organes, qui pondent leurs œufs chez les humains ou les animaux, qui pendant une certaine période se nourrissent de tissus vivants ou morts de l'hôte l'apparition de myiasis dans la cavité buccale peut être considérée comme une chose rare. Ce type de maladie touche le plus souvent les personnes de faible statut socio-économique, immunodéprimées, alitées âgées et souffrant de troubles psychiatriques. En raison de son grand potentiel destructeur, une prévention et un traitement appropriés opportuns et importants, il ya aussi peu de connaissances du professionnel dentaire pour le diagnostic et le traitement de cette pathologie, Pour cette raison, la présente étude rapporte un cas clinique de myiasis oral chez une personne âgée alitée avec une histoire de lésions malignes laryngée / glottale néoplasie, présentant la faiblesse

\footnotetext{
${ }^{1}$ Doctorant en sciences biomédicales à l'IUNIR- Instituto Universitário Italiano de Rosario - Argentine. Spécialiste en chirurgie maxillo-faciale et traumatologie par Ciodonto - Clinique dentaire intégrée. Diplômé en médecine dentaire de I'ASCES - Association Caruaruense de l'Enseignement Supérieur. Diplômé en Biologiste à I'UPE - Université de Pernambuco.

${ }^{2}$ Diplômé du cours de physiothérapie du Collège UNINASSAU.

${ }^{3}$ Diplôme en biologie UPE- Université de Pernambuco. Master en ressources naturelles - UFCG. Professeur à I'ONUPAR.
} 
physique et mentale et l'hygiène corporelle inadéquate et le manque de scellement des lèvres qui a conduit à l'infestation de larves dans la langue. Le diagnostic a été cliniquement établi basé sur l'observation du saignement de langue, du détachement de tissu, et de la présence des larves qui étaient entre les deuxième et troisième étapes du développement. Le traitement a été commencé comme hospitalisation du patient pour le débridement des tissus nécrotiques et l'enlèvement des larves et la prescription de l'ivermectine. Les patients alités présentant la faiblesse physique et métallique constituent un groupe de risque pour la myiase orale, et il est d'une grande importance de guider les soignants et les membres de la famille en ce qui concerne les soins dentaires afin de prévenir cette pathologie. Cependant, l'absence d'un consensus sur la meilleure approche thérapeutique pour les cas de myiase orale a été prise comme conduite thérapeutique pour la maladie l'enlèvement mécanique des larves et l'institution de l'utilisation orale de l'ivermectine.

Mots clés: Larve, myiasis humain, mouches, santé publique.

\section{INTRODUCTION}

La myiase est définie comme une pathologie dans laquelle il y a la peste des larves de mouches qui se nourrissent de tissus vivants ou morts (VINIT; JAYAVELU et SHRUTHA, 2013), bien que cette parasitose ait été décrite pendant de nombreuses années par des chercheurs, le terme technique pour désigner la bicheira a été suggéré en 1840 par le révérend F. W. Espoir dans son travail "On insects and their larvae occasionally found in the human body ", où il est proposé l'utilisation du terme "myiasis», qui est dérivé des mots grecs myia (mouche) et ase (maladie) (GUIMARAES; PAPAVERO et PRADO, 1983). Dans la littérature hindoue, la myiase a été considérée par la mythologie comme une punition des pécheurs (PARMAR; DAVESSAR et al., 2018).

Les patients atteints de myiase présentent généralement certains facteurs qui conduisent à la prédisposition à leur développement, tels que le retard mental, les troubles psychiatriques, la sénilité, les maladies vasculaires, les néoplasmes, le 
diabète et l'immunodépression et la consommation chronique d'alcool, et se produit de préférence chez les personnes âgées. (MARQUEZ; MATTOS et NASCIMENTO, 2007) (SILVEIRA; PINHEIRO et al., 2015) Cependant, les facteurs les plus pertinents pour le début de myiasis sont toujours l'occurrence des lésions exposées avec des exsudations et une mauvaise hygiène corporelle et buccale (NASCIMENTO ; OLIVEIRA et al., 2005) (TOURÉ, 1994).

II s'agit d'une situation de gravité mortelle pour le patient, en plus de causer la douleur et la destruction des tissus. La myiase buccale peut se présenter comme lacération de la cavité buccale, gonflement de la muqueuse buccale, inflammation du gingivale (RIBEIRO; PEREIRA et al.,2001). " ans le cas de Myiasis cavitaria, qui comprend les régions de la bouche et du nez et le sinus paranasal, on peut affirmer qu'il s'agit d'un type très rare de myiass " (BARNABÉ; FERRAZ et al., 2016). La variété la plus répandue d'espèces de mouches responsables de la plupart des cas de myiase comprend Cochliomyia hominivorax, Chrysomya bezziana, Oestrus ovis, Magnificent Wohlfahrtia et le Musca domestique. L'incidence de l'infection est rare même dans les pays en développement (KHAN; SABIR et al., 2018) (TOURÉ, 1994).

Le traitement de la myiase consiste essentiellement en l'élimination manuelle des larves, à l'aide d'une curette et d'une pince à épiler, généralement sous anesthésie ou analgésie, selon l'état clinique du patient, l'élimination des larves doit être effectuée avec soin, de sorte que le les larves ne sont pas fragmentées, et leurs restes restent en place, générant un processus infectieux (CAVALCANTI, 2008), bien qu'il existe sur le marché certains produits pouvant être utilisés pour faciliter l'élimination (BARNABÉ ; FERRAZ et al., 2016), la littérature rapporte avec un grand succès, l'utilisation systémique d'une dose d'ivermectine, un antibiotique macrolide semi-synthétique pour le traitement de cas plus graves impliquant l'existence d'une myiase buccale, s'avérant être une méthode sûre aux doses recommandées de 200 $\mu \mathrm{g} / \mathrm{kg}$. Même aux doses de 300 ou $400 \mu \mathrm{g} / \mathrm{kg}$, les patients sous traitement n'ont présenté aucun effet secondaire clinique important, à l'exception d'un prurit léger (GEALH ; FERREIRA et al., 2009) (RIBEIRO ; PEREIRA et al., 2001). 
Nous pouvons classer la myiase en trois types différents : biontophage ou primaire qui sont les larves diptères qui se développent sur ou à l'intérieur des vertébrés, se nourrissant des tissus de l'hôte vivant. Parmi ces espèces, nous pouvons trouver Cochliomy homini vorax et Dermatobia hominis. Les nécrobiolines secondaires sont celles dans lesquelles les larves de diptères se développent en matière organique qui est déjà décomcomposition (cadavres), agissant comme saprophages, mais peut également affecter les tissus nécrotiques dans un hôte vivant. Les genres les plus courants sont : Lucília, Phaenicia, Musca et Fannia. Pseudomyiasis (accidentelle) : cela peut se produire par ingestion d'ovules et/ou de larves de diptoran présents dans les aliments contaminés ingérés par l'hôte, causant un trouble intestinal (CAVALCANTI, 2008) (BOROS; BOROS et al., 2006).

\section{CAS CLINIQUE}

Un patient masculin de 69 ans diagnostiqué avec le néoplasme malin dans la langue (subissant la chimiothérapie) a été admis à la salle d'urgence de l'hôpital régional de Dom Moura dans Garanhuns - Pernambuco se plaignant de douleur et de seuant dans la région de bouche pendant plusieurs semaines, a été référé au service de chirurgie et de traumatologie de Bucomaxillofacial. À l'admission, le patient était afebrile, orienté et coopératif, le saignement a été observé dans la bouche, considérant le rapport de la famille que le patient a eu des problèmes dentaires, et des examens ont été exécutés. Dans l'évaluation intraorale a montré une lésion ulcéreuse bien définie avec le tissu nécrotique central et plusieurs larves vivantes sur la langue. (Figure 1) 
Figure 1 - Larves sur la langue.

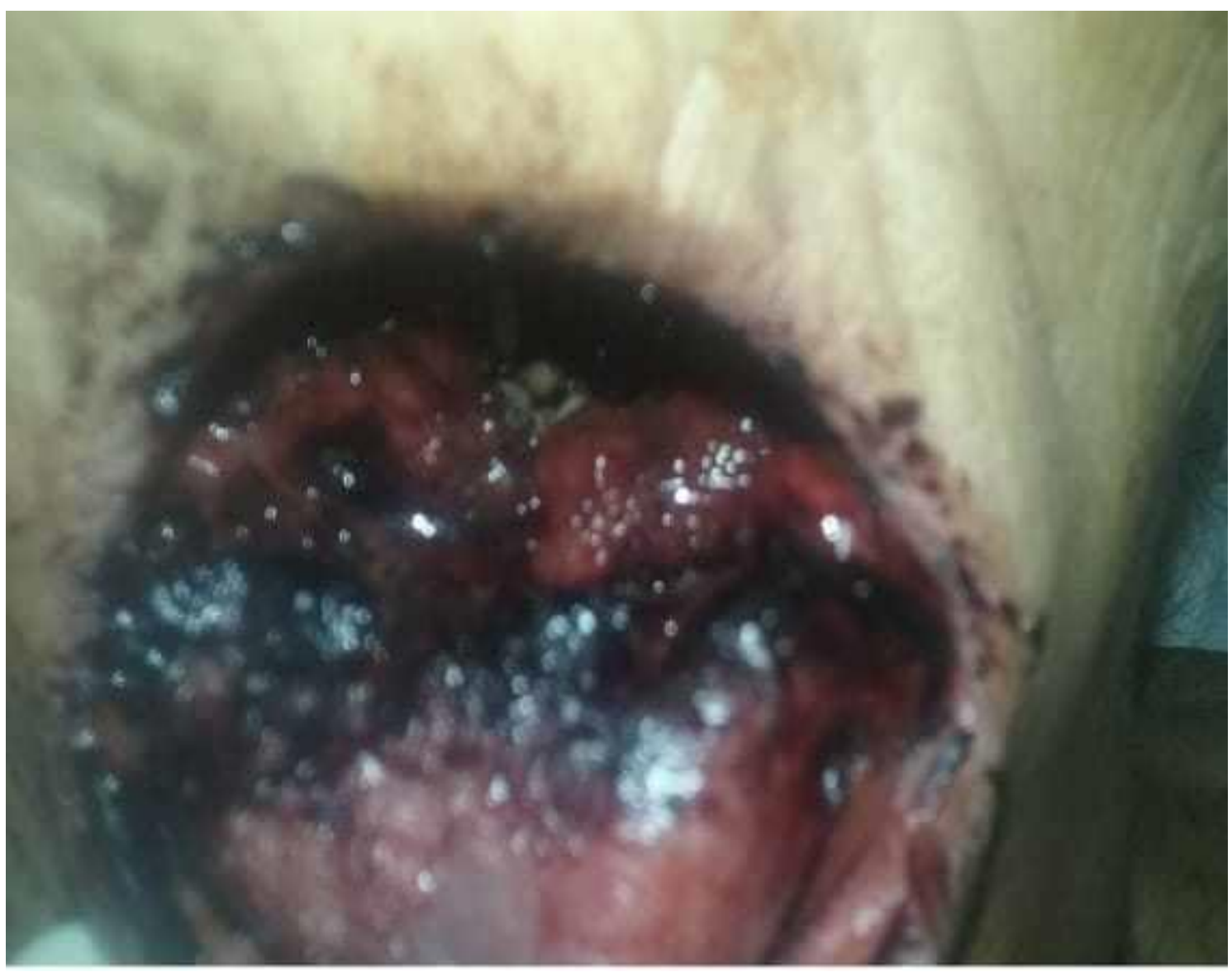

Source: Les auteurs (2020).

Le patient affecté par la maladie a présenté des facteurs prédisposants tels que la faiblesse physique et mentale, la déshydratation, l'hygiène corporelle inadéquate, le diabète, la malnutrition, la respiration buccale pendant le sommeil et le mauvais maintien de l'hygiène buccale.

Ainsi, le traitement symptomatique primaire a été exécuté. La plaie a été nettoyée et désinfectée avec une solution de chlorhexidine à 0,12\%. Les larves ont été enlevées manuellement sous anesthésie locale à l'aide de pinces cliniques, de l'ensemble du champ aseptique, les larves dans ce cas ont été recueillies et placées dans un plateau clinique. Environ 80 larves ont été retirées de la région, et toutes n'ont pas pu être enlevées (figure 2). 
Figure 2 - Larves retirées de la lésion

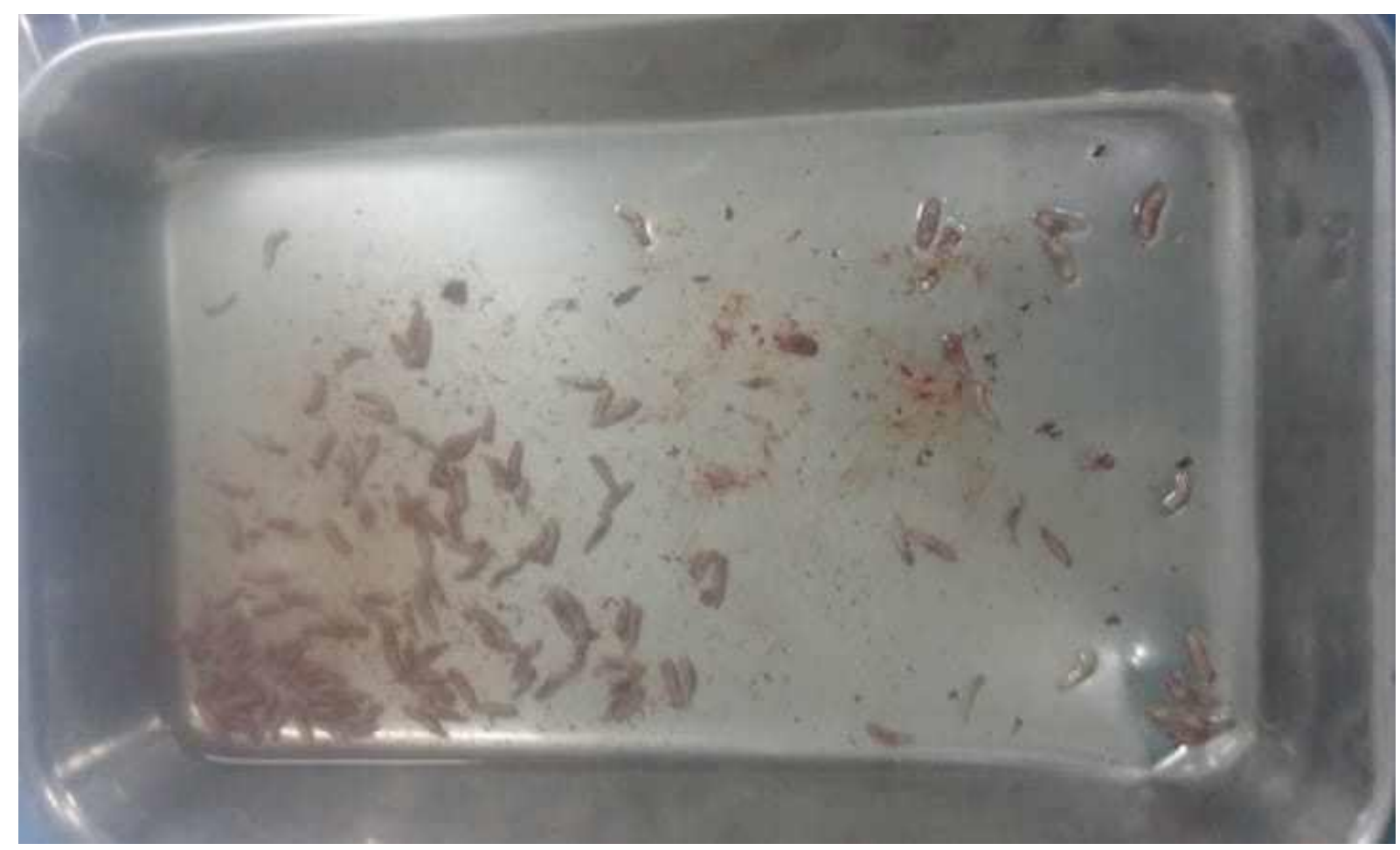

Source: Les auteurs (2020).

Le patient a été hospitalisé et la thérapie antibiotique intraveineuse a été commencée Cephalotin 1g, Dipirona et Ketoprofen, et une dose orale simple de Ivermectin deux comprimés, c.-à-d. 12 mg (approximativement $200 \mu \mathrm{g} / \mathrm{kg}$ ), après 10 jours toutes les larves restantes avaient été éradiquées, sans besoin de complémentation de Ivermectin.

\section{DISCUSSION}

Pendant le processus de développement des larves, les tissus adjacents présentent un processus inflammatoire, avec ou sans ulcération ou nécrose des tissus (CARVALHO; SANTOS et al., 2008). De nombreuses complications graves peuvent survenir en raison de la myiase : cellulite, érosion des os et des dents, bactériémie et décès (SZAKACS et MACPHERSON, 2007). 
Dans de nombreux articles consultés, nous avons observé que les auteurs mentionnent que la myiase orale est une pathologie rare, et que les diptrans n'ont aucune préférence pour l'âge, le sexe et l'ethnicité. Et que l'infestation de larve de dipterans est plus dans la zone rurale, infester les animaux domestiques tels que les chiens, chats, porcs, bœufs. L'opinion générale des auteurs est que les personnes infestées par les larves sont handicapées mentalement, les personnes ayant peu d'hygiène corporelle et buccale, comme un faible niveau d'éducation, les personnes de faible statut socio-économique (BOROS; BOROS et al., 2006).

Le diagnostic de myiase humaine est clinique, de sorte que l'on peut détecter certaines de ses complications peuvent être nécessaires pour effectuer la tomographie calculée, l'échographie ou l'imagerie par résonance magnétique (SHENOUDA; ENTEN et al., 2018).

La présence de larves sur la peau déclenche une réponse inflammatoire locale avec la migration et la prolifération des cellules inflammatoires telles que les neutrophiles, les mastocytes, les éosinophiles, les fibroblastes et les cellules endothéliales. Cependant, le nombre complet de cellules sanguines peut montrer des niveaux élevés de leucocytes et d'éosinophiles (PAYNE et COSGROVE, 1966) (SHENOUDA; ENTEN et al., 2018).

Certains auteurs préconisent un traitement conventionnel de la myiase, qui comprend l'élimination des larves à l'aide de substances chimiques, qui vise à promouvoir l'asphyxie des larves induisant leur sortie de la lésion. Dans les lésions plus grandes où il présente une image clinique de la cellulite adjacente, un débridement des tissus locaux serait indiqué avec l'enlèvement des tissus nécrotiques, mais d'autres défendent comme idéal, le traitement chirurgical, qui permet l'enlèvement de toutes les larves alliées à un débridement, et les antibiotiques pour prévenir l'infection secondaire, une autre méthode ont déjà été essayées comme l'utilisation d'une dose unique d'ivermectin (PARMAR; DAVESSAR et al., 2018). 
La myiase peut être considérée comme un problème de santé publique qui peut être évité en éliminant les facteurs de risque pour la santé humaine, tels que le manque d'assainissement de base et l'élimination inadéquate des déchets, où les matières organiques sont exposées, ce qui attire les insectes et les petits animaux, créant un cycle durable de la saleté. Le mauvais assainissement est probablement le facteur de risque le plus important pour la myiase humaine (FRANCESCONI et LUPI, 2012).

\section{CONCLUSION}

Dans la littérature, plusieurs traitements pour la myiase cavitaire ont été proposés, allant de l'extraction mécanique à l'utilisation de substances topiques, orales et intraveineuses. Mais un diagnostic tôt et correct permet un traitement avec un pronostic favorable. Toutefois, quels que soient les moyens employés, il est essentiel que le patient soit orienté vers l'adoption de mesures d'hygiène personnelle appropriées. Cependant, il n'y a pas de consensus sur la meilleure approche thérapeutique pour les cas de myiase orale.

\section{RÉFÉRENCES}

BARNABÉ, A. S. et al. Epidemiologia Da Miíase Cutânea: Revisão Da Literatura. Atas de Ciências da Saúde, São Paulo, v. 4, n. 2, p. 14-22, Abril - Junho 2016.

BOROS, L. F. et al. Míase Labial. Revista Dens, v. 14, n. 1, p. 1-10, Revista Dens, v.14, n.1, maio/outubro 20062006.

CARVALHO, R. W. F. et al. Oral and maxillofacial myiasis associated with epidermoid carcinoma: a case report. Journal of Oral Science, v. 50, n. 1, p. 103-105, 2008.

CAVALCANTI, A. L. Mí́ase Oral: etiologia, diagnóstico e tratamento. Rev. Fac. Odontol. Porto Alegre, Porto Alegre, v. 49, n. 2, p. 32-35, maio/ago. 2008. 
CAVALCANTI, A. L. Miíase Oral: etiologia, diagnóstico e tratamento. Rev. Fac. Odontol. Porto Alegre, Porto Alegre, v. 46, n. 2, p. 32-35, maio/ago. 2008.

FRANCESCONI, ; LUPI,. Myiasis. Clinical Microbiology Reviews , v. 25, n. 1, p. 79 105, Jan. 2012.

GEALH, W. C. et al. Treatment of oral myiasis caused by Cochliomyia hominivorax: two cases treated with ivermectin. British journal of Oral and Maxillofacial Surgeons, v. 47 , n. 1, p. 23-26, January 2009.

GUIMARAES, J. H.; PAPAVERO, ; PRADO, A. P. D. As Mi1ases Na Região Neotropical. Revista Brasileira De Zoologia (Identificação, Biologia, Bibliografia), São Paulo, v. 1, n. 4, p. 239.416, 1983.

KHAN, B. A. et al. Oral and cutaneous myiasis in a 5 year old child from Karachi, Pakistan. Le Infezioni in Medicina, v. 4, p. 385-388, 2018.

MARQUEZ, A. T.; MATTOS, M. D. S.; NASCIMENTO, S. B. Miíases associadas com alguns fatores sócio-econômicos em cinco áreas urbanas do Estado do Rio de Janeiro. Revista da Sociedade Brasileira de Medicina Tropical, v. 40, n. 2, p. 175180, março - abril 2007.

NASCIMENTO, E. M. F. D. et al. Miíases Humanas Por Cochliomyia Hominivorax (Coquerel, 1858) (Diptera, Calliphoridae) Em Hospitais Públicos $\mathrm{Na}$ Cidade Do Recife, Pernambuco, Brasil. Entomología y Vectores, v. 12, n. 1, p. 37-51, 2005.

PARMAR, S. et al. Tracheostomal Myiasis: A Case Report And Review Of Literature. International Journal of Scientific Research, v. 7, n. 12, p. 4-6, December 2018.

PAYNE, J. A.; COSGROVE, G. E. Tissue changes following Cuterebra infestation in rodents. The American Midland Naturalist, v. 75, n. 1, p. 205-213, Jan. 1966.

RIBEIRO, F. A. Q. et al. Tratamento da miíase humana cavitária com ivermectina oral. Rev Bras Otorrinolaringol, v. 67, n. 6, p. 755-761, nov./dez. 2001. 
SHENOUDA, M. et al. Human Botfly: A Case Report and Overview of Differential Diagnosis. J Investig Med High Impact Case Rep., v. 6, Jan-Dec; 2018.

SILVEIRA, M. A. A. et al. Cavitary myiasis mimicking peritonsilar abscess. Brazilian Journal of Otorhinolaryngology, v. 81, p. 336-338, 2015.

SZAKACS, T. A.; MACPHERSON,. Nosocomial myiasis in a Canadian intensive care unit. CMAJ, v. 177, n. 7, p. 719-720, 25 September 2007.

TOURÉ, S. M. Les myiases d'importance économique. Rev. sci. tech. Off. int. Epiz., 1994, 13 (4), 1053-1073, v. 13, n. 4, p. 1053-1073, 1994.

VINIT, G. B. ; JAYAVELU, P.; SHRUTHA, S. P. Oral myiasis in a maxillofacial trauma patient. J Pharm Bioallied Sci., v. 5, n. 6, p. 195-197, 5 Jul 2013.

Envoyé : Février 2020.

Approuvé : Août 2020. 